\title{
Cardiac MRI: Standardized Right and Left Ventricular Quantification by Briefly Coaching Inexperienced Personnel
}

\author{
Niek H. Prakken*, ${ }^{1}$, Birgitta K. Velthuis ${ }^{1}$, Evert-Jan J. Vonken ${ }^{1}$, Willem P. Mali ${ }^{1}$ and \\ Maarten-Jan J. Cramer ${ }^{2}$ \\ ${ }^{1}$ Department of Radiology, University Medical Center Utrecht, Heidelberglaan 100, 3584 CX, Utrecht, the Netherlands
${ }^{2}$ Department of Cardiology, University Medical Center Utrecht, Heidelberglaan 100, 3584 CX, Utrecht, the Netherlands
}

\begin{abstract}
MRI quantification of the cardiac ventricles is time-consuming, especially for the right ventricle due to its complex geometry. Using a short axis MRI protocol, we tested if briefly coaching persons inexperienced in cardiac MRI provides reliable right and left ventricular quantification.

22 healthy subjects (mean age $26 \pm 4.2$ years) underwent short-axis breath-hold SSFP sequence cardiac MRI. Two persons inexperienced in cardiac MRI independently traced endocardial and epicardial contours of both ventricles with a predefined contour tracing protocol. Measurements were repeated after visual correction of each two most basal slices of the endocardial contours and epicardial contours in the learning curve. Five random short-axis cines were retraced for intraobserver variability. Measurements were performed blinded within 4 months. Agreement was assessed with the BlandAltman method.
\end{abstract}

No systematic bias was observed and measurements were within acceptable limits of agreement after brief coaching. Repeat measurements following visual correction significantly improved inter-observer differences, especially for mass calculations. Maximum interobserver and intraobserver disagreement of the final protocol were respectively $\leq 8 \%$ and $\leq 5 \%$.

Brief coaching of persons inexperienced in cardiac MRI, using the short axis MRI protocol, provides reliable volume, function and mass quantification of both ventricles.

\section{INTRODUCTION}

Cardiac MRI (CMR) is increasingly requested by clinicians worldwide in the workup of patients with suspicion of or established diagnosis of cardiovascular disease. The accuracy and reproducibility of CMR is well known [1-5].

Quantitative volume, mass and function measurements are often mandatory for making an accurate diagnosis [1]. Left ventricle (LV) quantification is an established clinical tool, but accurate right ventricle (RV) dimensions and function are also increasingly used for the workup and follow-up in congenital heart disease and cardiomyopathies such as arrhythmogenic RV cardiomyopathy (ARVC) [6, 7]. The short-axis multi-slice acquisition is an important part of standardized clinical cardiac MRI protocols and shows good correlation with in vivo standards and radionuclide angiography, making it well suited for the determination of quantitative ventricular parameters $[2,8]$.

Automatic segmentation is often insufficient if more accurate quantification is required due to varying inclusion of trabeculae and papillary muscles of the LV, and varying inclosure of the most basal slice and the LV outflow tract (LVOT). The non-geometrical shape and asymmetric basal appearance of the RV outflow tract (RVOT) and tricuspid valve (TV) makes RV tracing difficult [2, 9-12].

*Address correspondence to this author at the Department of Radiology, University Medical Center Utrecht, Heidelberglaan 100, 3584 CX, Utrecht, the Netherlands; E-mail: n.prakken@umcutrecht.nl
A robust and reproducible contour tracing protocol is needed to measure LV and RV volume, function and mass quantitatively $[9,10,13,14]$. No clear instructions are available for defining how to quantify volumes and wall mass by contour tracing the LV and RV. Existing RV protocols show high intra- and interobserver variability (up to 60\%) for the two most basal endocardial and epicardial contours and RV wall mass is especially difficult to reproduce $\left(\mathrm{R}^{2}\right.$ equalling 0.85 ) $[2,5,9-11,15-17]$.

In this study we aimed to establish a robust short axis cine-based contour tracing protocol for both $\mathrm{LV}$ and RV which can be used to train persons inexperienced in cardiac MRI.

\section{METHODS}

\section{Study Population}

22 healthy subjects (mean age $26 \pm 4.2$ years) underwent MRI imaging. No cardiac disease or valvular pathology was visualized during the MRI scan in any of the subjects. The absence of pathology was also confirmed by an elaborate questionnaire, blood pressure measurement, electrocardiography, and echocardiography. This study complies with the Declaration of Helsinki. The research protocol was approved by the Institutional Ethics Committee University Medical Center Utrecht, and written informed consent has been obtained from all subjects. 


\section{Acquisition Protocol}

Cardiac MRI images were obtained with a 1.5-T Achieva MRI scanner (Philips, Best, the Netherlands) with a phasedarray cardiac coil. An ECG-gated breathhold vertical long axis (2 chamber) LV and horizontal long axis (4 chamber) image were used to identify the cardiac short axis. The whole heart was imaged in the short axis plane, from ventricular apex to base including both atria, using 14 to 20,10 $\mathrm{mm}$ slice steady-state free precession (SSFP) cines without interslice gap, of 50 frames per cardiac cycle, matrix 256x256, and FOV 350-400 [18].

All images were acquired during 10- to 15 -second breathholds and stored digitally for offline analysis of cardiac volumes, mass and function. All CMR scans were performed by the same experienced operator.

\section{Image Analysis}

Analysis was performed on a clinical workstation with semi- automated contour tracing software (View Forum cardiac package version R5.1V1L1 2006, Philips, Best, the Netherlands). Two persons inexperienced in cardiac MRI (medical students) independently traced endocardial (for volume measurement) and epicardial (for wall mass defined as ventricular wall enclosed by endocardial and epicardial contours) contours of both ventricles with the predefined contour tracing protocol in 22 subjects. Measurements were repeated after visual correction of the endocontours of each two most basal ventricular slices and all epicardial contours as a learning curve. For inter-observer variability measurement, all 22 subjects were retraced. Each observer subsequently retraced 5 random short-axis cines for intra-observer variability. All measurements were performed blinded and at random over a period of 4 months. The level of agreement was assessed utilizing the statistical regression model and the Bland-Altman method [1, 2, 19-21].

\section{Contour Tracing Protocols}

We adapted a contour tracing protocol to calculate left and right ventricular volumes and wall mass based on one already used in the Radiology department $[19,22]$. In this protocol both LV and RV were traced from the most apical short-axis slice to the most basal slice on the ventricular side of respectively the tricuspid valve and mitral valve. The LV and RV end-diastolic (EDV) and end-systolic (ESV) volumes, derived ejection fractions (EF), stroke volumes (SV) cardiac outputs (CO), and end-diastolic masses (EDM) were calculated by adding the areas for each slice per ventricle, multiplied by the slice thickness using the Simpson's rule [3, 5]. No inter-slice gaps are present in our short-axis protocol.

The quantitative analysis included two steps for both left and right ventricle: 1 . tracing the endocardial contour in enddiastolic and end-systolic phase for volume and function; 2. the epicardial border in end-diastolic phase for wall mass.

\section{Left Ventricle}

First, the LV endocardial contours were traced, starting with the determination of the end diastolic phase (mitral valve has just closed). The endocardial contours were traced from the most apical to the most basal slice excluding the papillary muscles and trabeculae. The papillary muscles and trabeculae were excluded from the endocardial contour and therefore included in the blood volume.

The endocardial contour forms a smooth ellipsoid line between the trabeculae and the endocardial border (Fig. 1). The most basal LV slice had to show at least $50 \%$ visible myocardial circumference at mitral valve level to be included (Fig. 2). The LV outflow tract is also included in the endocardial contour, with the lateral border of the aortic valve as a straight line alongside the aortic valve plane (Fig. 1F, 2B).

Second, the epicontours were traced at end diastole, including ventricular septum for LV mass measurement and overlapping the endocontour at valve planes (Fig. 1ABC).

\section{Right Ventricle}

The RV endocardial contours were traced with instructions to accommodate for the difficulties encountered in drawing contours for the most basal two slices.

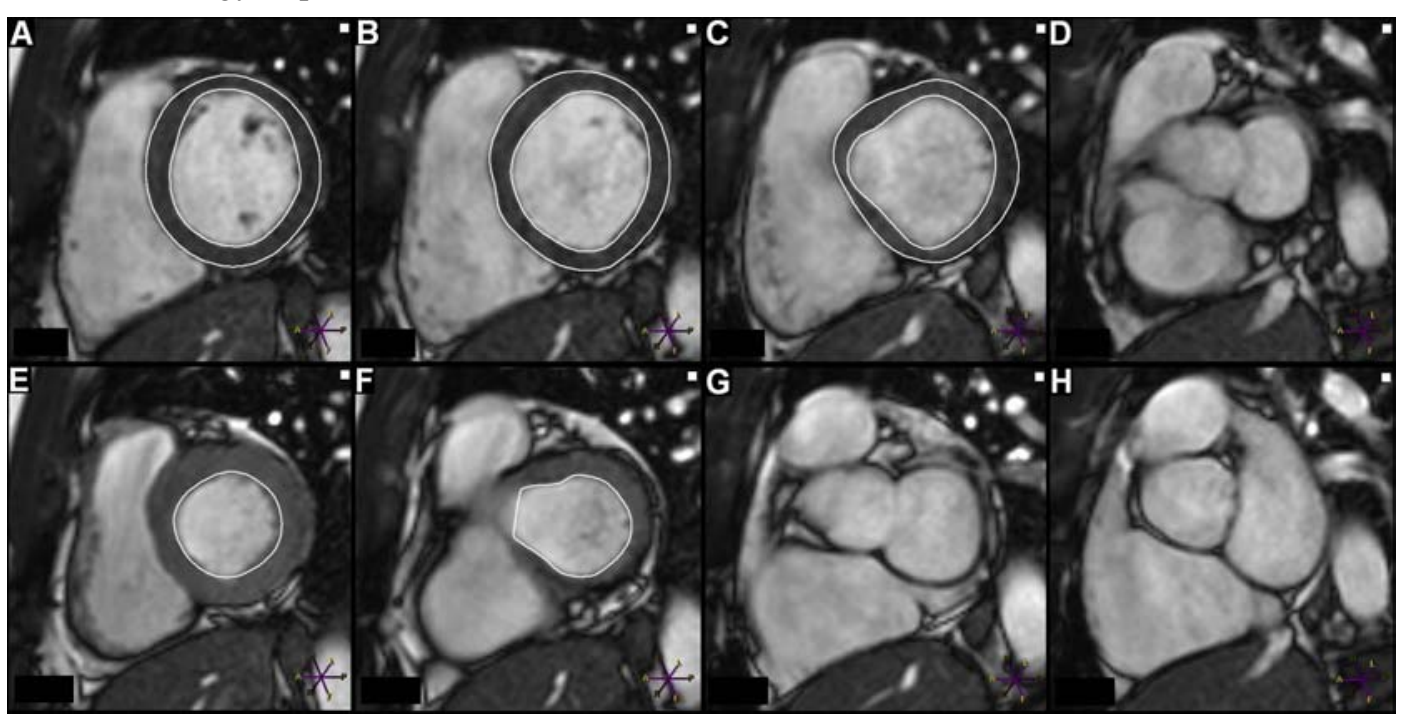

Fig. (1). Basal slices in end-diastole (A, B, C, D); and end-systole ( $\mathbf{E}, \mathbf{F}, \mathbf{G}, \mathbf{H})$ in one subject. Because the ventricle shortens during contraction the most basal end-diastolic ventricular slice $(\mathbf{C})$ is not at the same level as the most basal end-systolic slice $(\mathbf{F})$. 


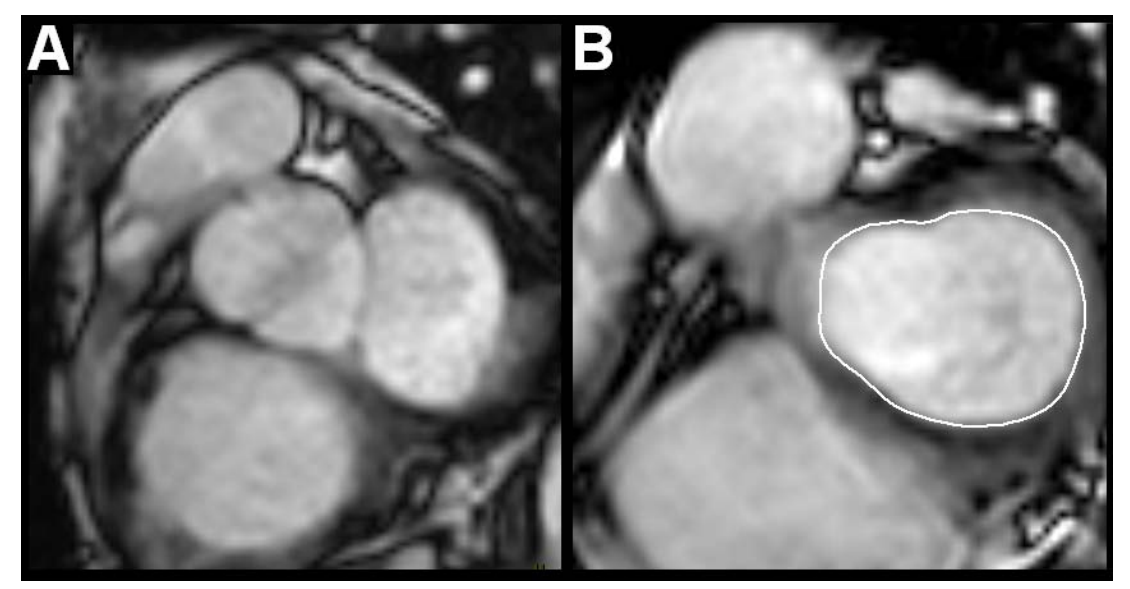

Fig. (2). Determining the amount of visible LV myocardial circumference. A: in this subject's end-diastolic phase, less than $50 \%$ myocardial circumference is visible, therefore no endocontour is drawn here; $\mathbf{B}$ : in this subject's end-diastolic phase, over 50\% myocardial circumference is visible, and therefore included in the endocardial contour.

\section{Instructions for Tricuspid Valve (TV) Tracing}

1. As the TV opens into the RV in diastole, the TV can be visible in more than one basal slice. The TV can only be excluded once on the most basal slice at enddiastole where it is at least $50 \%$ visible in the RV (Fig. 3).

2. If the upper TV border is clear, the TV is fully excluded from the most basal slice at end-diastole (Fig. 3A).

3. When the upper half of the TV border is unclear, the lower half is drawn in with a straight cut-off line between lateral border and septum (Fig. 4C).

4. In the most basal end-diastolic slice, a RV contour can only be drawn if visible for at least 3 phases in diastole (Figs. 3, 4).
5. In the most basal end-systolic slice, the visible RV contour is always traced because, at this point in the cardiac cycle, the heart is in maximum longitudinal contraction (Fig. 4E).

\section{Instructions for Tracing the Pulmonary Valve (PV)}

The PV can only be left out the 2 most basal slices that are used for drawing the endocardial contour.

The border of the valve (PV plane) forms a straight line. This level is determined as where the muscular ventricle border is no longer visible and a bulge appears from the valve annulus. No contractions are visible above the PV plane (Fig. 3A, 4C).

The papillary muscles, trabeculae and moderator band are excluded from the endocontour and included in the blood volume.

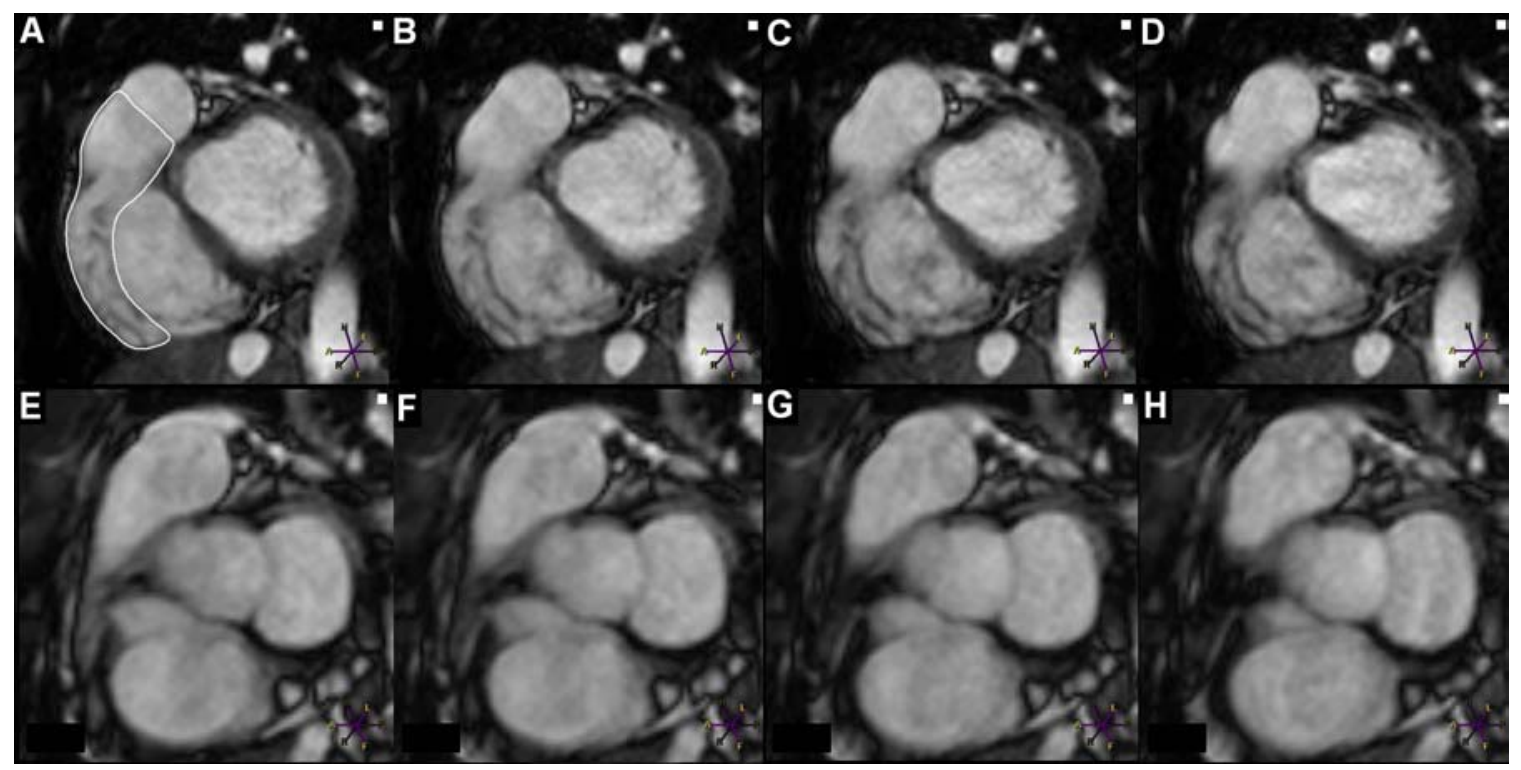

Fig. (3). ABCD: in this subject the first four phases (A: phase 1; B: phase 2; C: phase 3; D: phase 4) starting with the end-diastolic phase (A) are shown. Since the RV trabecularisation around the TV stayed visible for more than the required 3 phases, it was included in the endocardial contour together with the RV outflowtract. Anatomical borders of the TV and PV were clear. EFGH: four phases (E: phase 1; F: phase 2; G: phase 3; H: phase 4) starting in end-diastolic phase (E) of another subject are shown here. Since the RV trabecularisation around the TV stayed visible for less than 3 phases, it was left out the endocardial contour. 


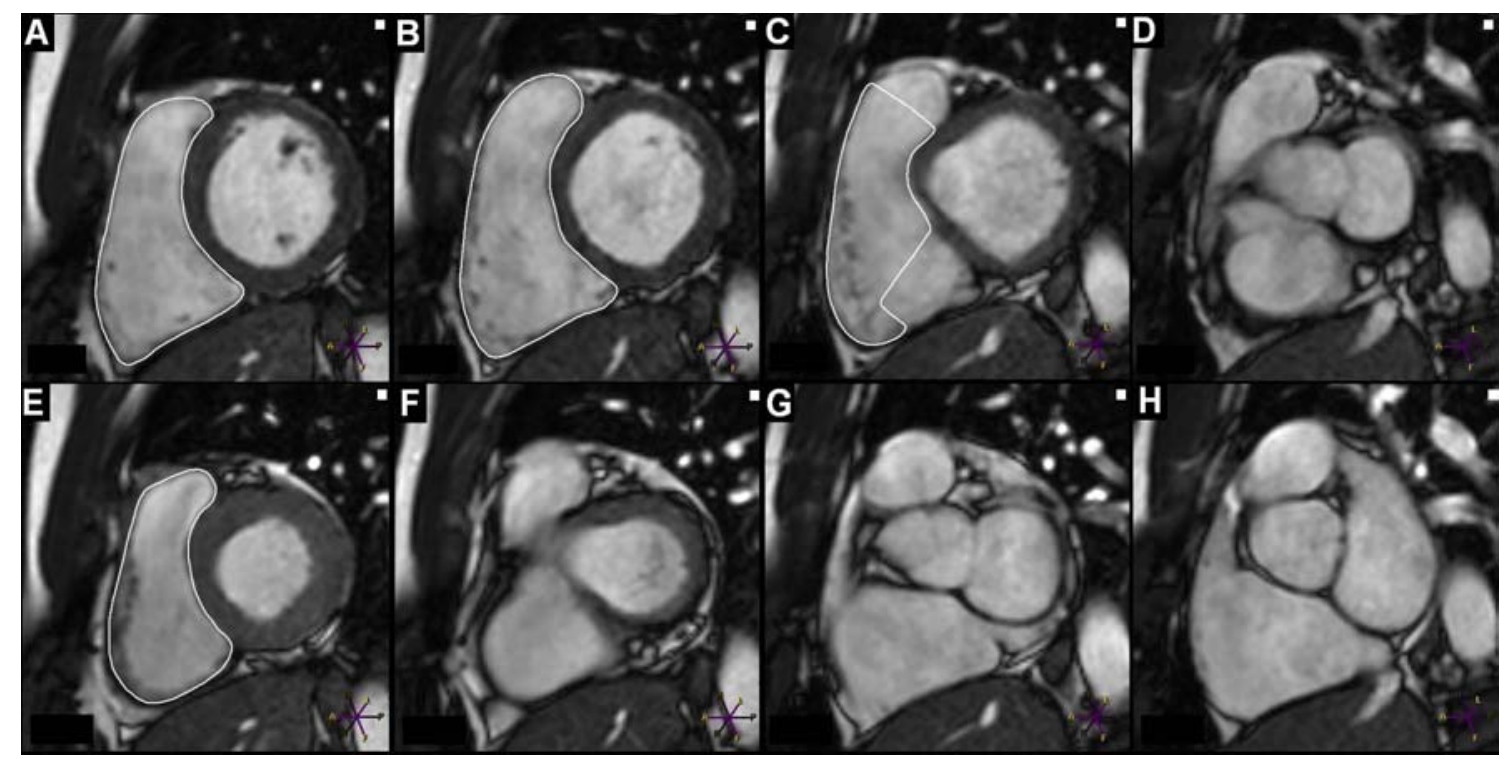

Fig. (4). Basal slices in end-diastole $(\mathbf{A}, \mathbf{B}, \mathbf{C}, \mathbf{D})$; and end-systole $(\mathbf{E}, \mathbf{F}, \mathbf{G}, \mathbf{H})$ in one subject. A straight cut-off line is used for the TV because the upper border of the TV with the RV is unclear $(\mathbf{C})$.

The RV epicontours are traced at end-diastole (phase 1). The epicontour overlaps both the septal part of the endocontour, and the endocontour borders at valve planes. Visible RV myocardium should be included in the epicontour. However, the RV wall is usually so thin that the lateral border of the RV consists mostly of an artefact (dashed black-and-grey line) in normal healthy subjects, and the epicontour has to be traced just outside this artefact (Fig. 5).

The above described instructions solved most issues of how to trace the two most basal RV short axis slices. However, in a few cases anatomical borders remained unclear and the "Linking" tool on the View Forum station was used. With this tool the vertical long axis ( 2 chamber) LV, horizontal long axis (4 chamber), vertical long axis ( 2 chamber)
RV, LVOT, and RVOT cines were phase and slice linked to the short axis cines (Fig. 6). Ventricular "cut-off" lines were drawn between the most lateral and most medial point on each annular ring of each valve in every view. This way the observer determined if a certain point at end-diastole or at end-systole on a short-axis slice fell within one of the "cutoff" lines on one or more of the other linked cine views. If the point was located at the ventricular side of the "cut-off" line, this part was taken inside the endocontour. To illustrate this, four points in space and time are shown on the short axis series (marked A through D). Each crosshair corresponds with the same point in space and time on one or more of the other series (Fig. 6).

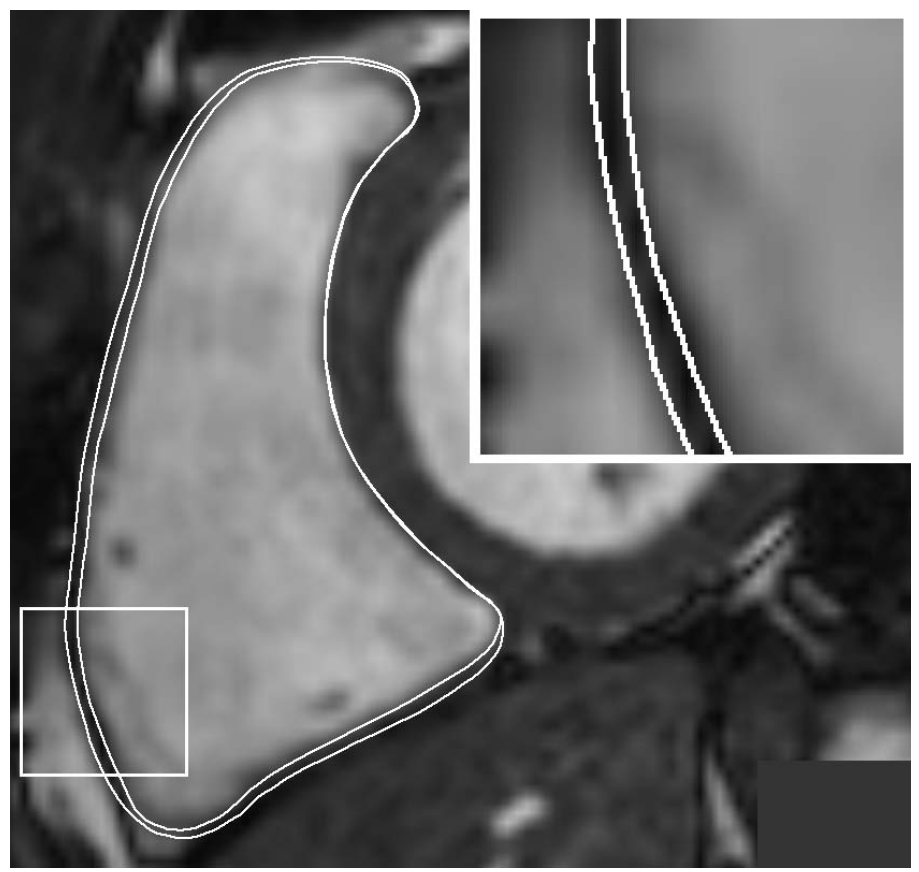

Fig. (5). Delineation of the RV epicontour. 


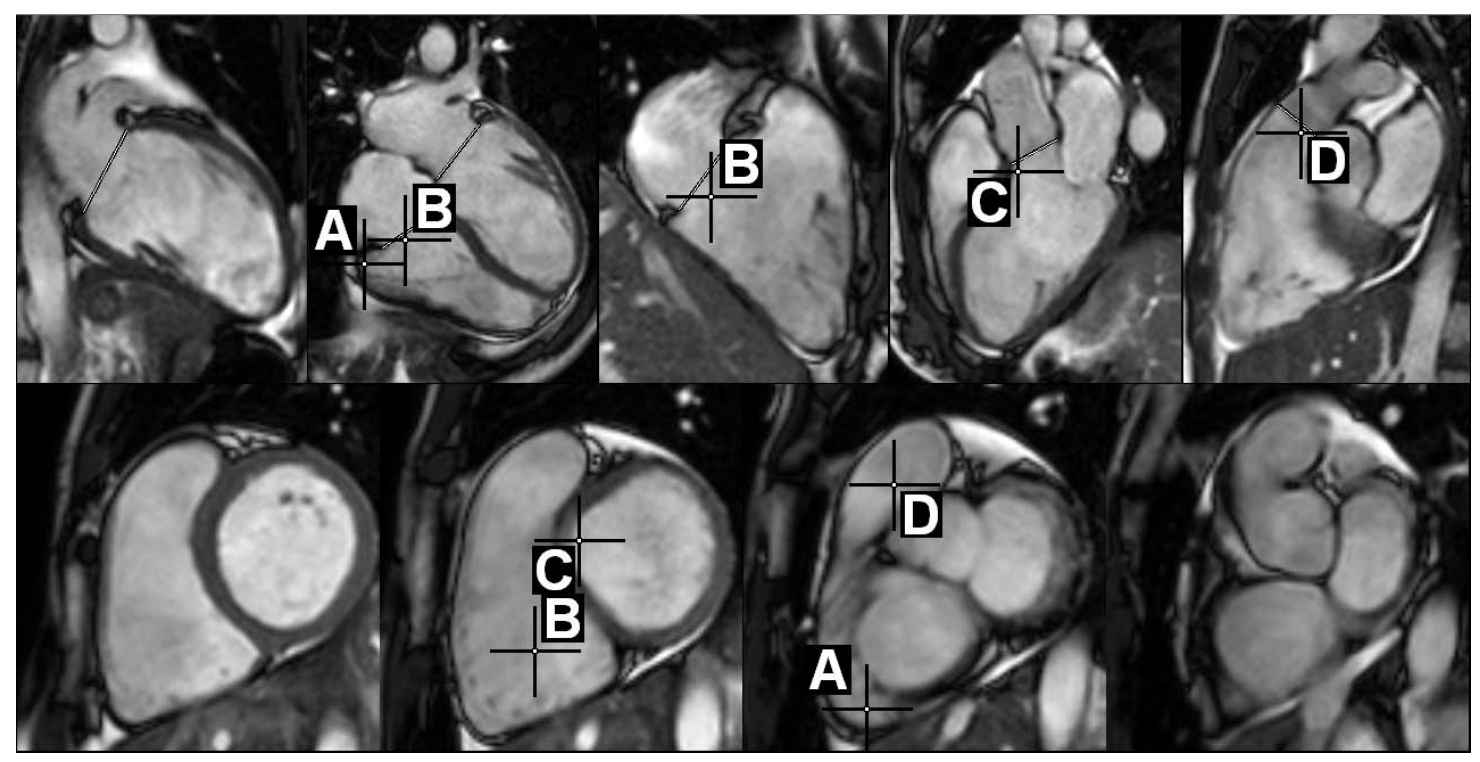

Fig. (6). Using the linking tool to link the short axis cine to the LVOT, RVOT, 2 chamber left and right, and 4 chamber cines to visualize the anatomical borders of the ventricles. From left to right upper row: vertical long axis ( 2 chamber) left ventricle; horizontal long axis (4 chamber); vertical long axis ( 2 chamber) right ventricle; LVOT; RVOT- cines with predefined delineations in black by using the linking tool. Crosshairs A through D: each crosshair demarcates a point in space on the short axis cine (lower row) which corresponds with the crosshair on one or more of the other views.

\section{Statistical Analysis}

All ventricular volumes, functions and masses were expressed as mean \pm standard deviation (mean \pm SD). The levels of agreement in measured values were evaluated with the Bland-Altman analysis by calculating the bias (mean differ- ence) and the $95 \%$ limits of agreement (2 SD around the mean difference). The difference between observer values was plotted against their mean to avoid artificial trends. The relationship between the two independent observers was evaluated with linear regression analysis with Pearson's cor-

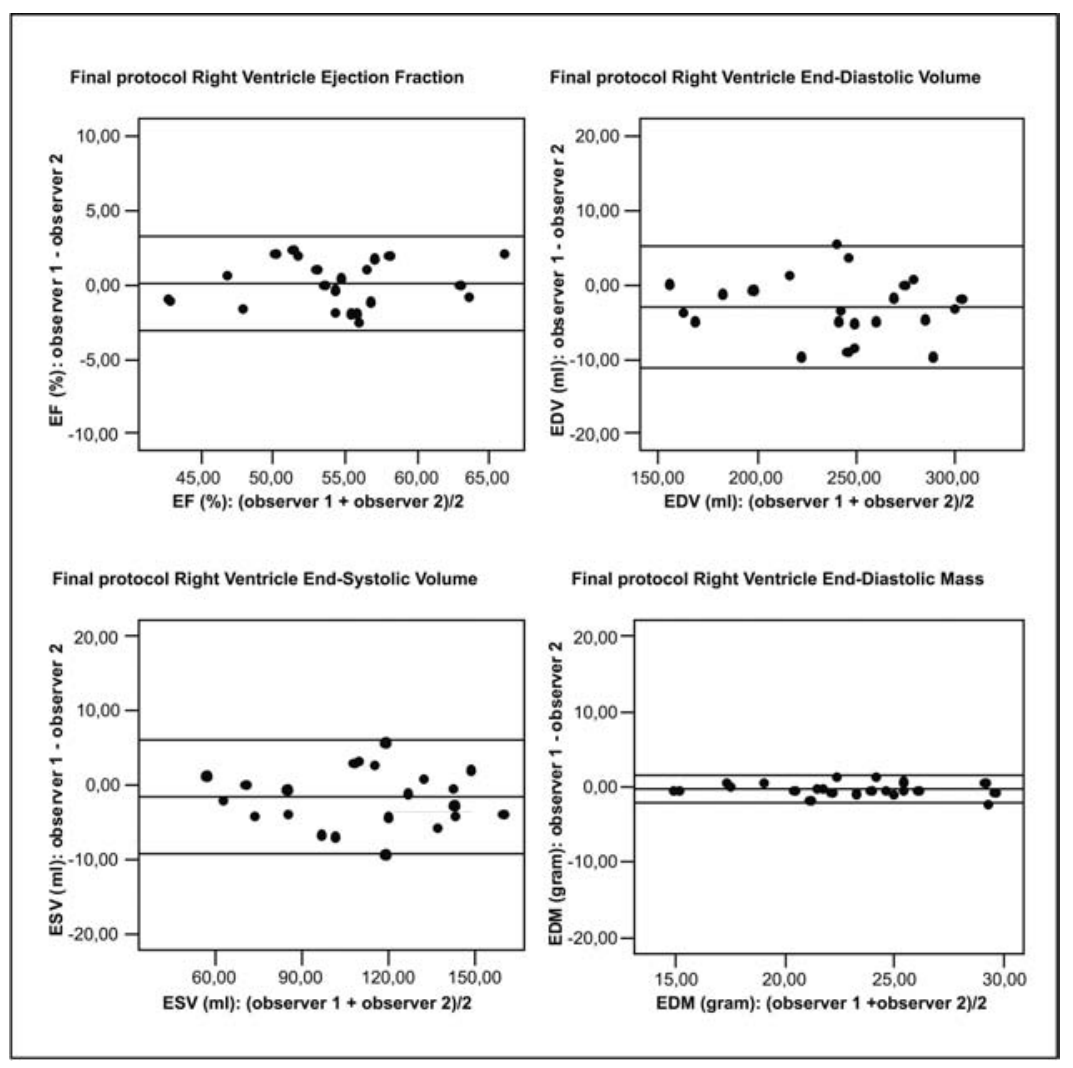

Fig. (7). Final protocol inter-observer Bland Altman Analysis. 
Table 1. Reproducibility of Results with Maximum Disagreement Values

\begin{tabular}{|c|c|c|c|c|c|c|}
\hline \multirow[b]{2}{*}{ Parameter } & \multirow{2}{*}{$\begin{array}{c}\text { Inter-OITP }(\mathbf{n}=\mathbf{2 2}) \\
\text { MD \% }\end{array}$} & \multicolumn{3}{|c|}{ Inter-OATP $(n=22)$} & \multicolumn{2}{|c|}{ Intra-OATP $(n=10)$} \\
\hline & & MD \% & Mn-D (sd) & $\mathbf{R}^{2}$ - Linear & Mn-D (sd) & MD \% \\
\hline LV EF & 8 & 5 & $-0.69(0.99)$ & 0.954 & $0.29(0.92)$ & 4 \\
\hline LV SV & 8 & 8 & $-2.6(3.36)$ & 0.974 & $0.42(3.46)$ & 5 \\
\hline LV EDV & 6 & 3 & $-2.1(3.56)$ & 0.990 & $-0.71(4.81)$ & 4 \\
\hline LV ESV & 7 & 5 & $0.52(2.06)$ & 0.990 & $-1.16(3.01)$ & 5 \\
\hline LV EDM & 35 & 8 & $0.67(3.42)$ & 0.988 & $1.14(2.95)$ & 5 \\
\hline RV EF & 8 & 5 & $0.09(1.57)$ & 0.933 & $-0.28(1.15)$ & 3 \\
\hline RV SV & 8 & 7 & $-1.35(4.92)$ & 0.943 & $0.00(0.00)$ & 5 \\
\hline RV EDV & 6 & 4 & $-2.95(4.10)$ & 0.991 & $0.14(3.51)$ & 3 \\
\hline RV ESV & 17 & 8 & $-1.6(3.85)$ & 0.983 & $0.90(2.87)$ & 4 \\
\hline RV EDM & 71 & 8 & $-0.2(0.89)$ & 0.958 & $-0.14(0.42)$ & 3 \\
\hline
\end{tabular}

OITP = observer in training period; OATP = observer after training period; MD $\%=$ maximum disagreement percentage; Mn-D = mean difference; LV = left ventricle; RV = right ventricle; $\mathrm{EF}=$ ejection fraction; $\mathrm{SV}=$ stroke volume; $\mathrm{EDV}=$ end-diastolic volume; $\mathrm{ESV}=$ end-systolic volume; $\mathrm{EDM}=\mathrm{end}$-diastolic mass.

relation coefficient. The significance of biases was tested through the use of paired t-tests with a 2-sided alternative. Values of $\mathrm{P}<0.05$ were considered significant. Measurement of reproducibility was evaluated by calculating the intraobserver and interobserver variability of each technique, defined as the absolute difference between the corresponding repeated measurements expressed in percent of their mean.

\section{RESULTS}

No systematic bias was observed and measurements were within acceptable limits of agreement after brief coaching. Interobserver differences improved significantly, especially for mass calculations, after adjustment of the initial protocol (Fig. 7, Table 1). Maximum inter and intraobserver disagreement in all cases of the final protocol was respectively $\leq 8 \%$ and $\leq 5 \%$, with $\mathrm{R}^{2}$-Linear regression coefficients equal to or greater than 0.933 for inter observer correlation. LV versus RV stroke volume had a $\mathrm{R}^{2}$-Linear interobserver correlation of 0.908 and $\mathrm{R}^{2}$-Linear intraobserver correlation of 0.933, showing good correlation of LV and RV stroke volumes.

\section{DISCUSSION}

No clear instructions are available for defining how to quantify volumes and wall mass by contour tracing the LV and RV. We adapted a widely used short axis contour tracing protocol with a specific set of instructions for the most basal planes to obtain good reproducibility and correlation of LV and RV results that can be used not only for coaching, but also for the standardization of automatic or semi-automatic segmentation, particularly for the RV [23].

Following brief coaching how to perform the measurements, persons inexperienced in cardiac MRI can achieve reliable results.

Our protocol can be especially beneficial in quantification of RV volumes and mass for possible cardiomyopathy with RV involvement, like ARVC, and follow-up of congenital heart disease such as tetralogy of Fallot [24-28].

Steen et al. (2007) studied factors influencing volume and mass measurement and stated that objective criteria for defining the precise blood-pool myocardial tissue interface are needed [29]. Previous studies reported large variability in inter- and intraobserver differences for endocardial and epicardial contours in the two most basal planes $[2,5,9-11$, 15-17]. In addition, exact instructions on what to include in the most basal planes to calculate volumes and wall mass are often unclear $[12,20,30]$.

The LVOT is included in the endocardial contour in most reported LV protocols [20,30]. We also chose to include the LVOT and RVOT as the outflow tract is part of the ventricles and these clear anatomical landmarks increase reproducibility. Including the outflow tracts will increase the ventricular volumes. This will not affect SV (EDV-ESV) but may reduce EF (ESV divided by EDV) [31-33].

The amount of papillary muscle or trabeculae which can be drawn in for mass varies substantially and can make up to $20 \%$ difference in wall mass [12, 20, 34-37]. We therefore chose not to include papillary muscles in the wall mass. Instead, we included papillary muscles and trabeculae in the blood volume for efficiency and reproducibility [29].

We used the short axis cine as it is part of the standard MRI scan protocol in most clinical laboratories, and published articles routinely calculate ventricular volumes by applying the method of disks to stacks of short-axis images (Simpson's rule) [1] Some authors claim that the radial long axis has better reproducibility as the anatomical landmarks are clearer on those views. [9, 15]. In subjects in which we encountered difficulties due to lack of anatomical landmarks, especially for the TV, we established "cut-off" lines that correspond with the valve planes on the RVOT, LVOT, 4 chamber and 2 chamber left and right cines. We used SSFP because it shows better blood-to-myocardium contrast and 
higher reproducibility of RV in comparison to Turbo Gradient Echo. A limitation of our study is that reproducibility was not tested in a different set of patients after the initial coaching. It remains important that the reporting physician checks results of the persons performing quantification.

\section{CONCLUSIONS}

Brief coaching using an adapted short axis cardiac MRI protocol with specific instructions, provides reproducible volume, function and mass quantification of the RV and LV to facilitate cardiac MRI reporting.

\section{REFERENCES}

[1] Sugeng L, Mor-Avi V, Weinert L, et al. Quantitative assessment of left ventricular size and function: side-by-side comparison of realtime three-dimensional echocardiography and computed tomography with magnetic resonance reference. Circulation 2006; 114 : 654-61.

[2] Shors SM, Fung CW, Francois CJ, Finn JP, Fieno DS. Accurate quantification of right ventricular mass at MR imaging by using cine true fast imaging with steady-state precession: study in dogs. Radiology 2004; 230: 383-88.

[3] Baldy C, Douek P, Croisille P, Magnin IE, Revel D, Amiel M. Automated myocardial edge detection from breath-hold cine-MR images: evaluation of left ventricular volumes and mass. Magn Reson Imaging 1994; 12: 589-98.

[4] Plein S, Smith WH, Ridgway JP, et al. Measurements of left ventricular dimensions using real-time acquisition in cardiac magnetic resonance imaging: comparison with conventional gradient echo imaging. MAGMA 2001; 13: 101-8.

[5] Hergan K, Schuster A, Fruhwald J, Mair M, Burger R, Topker M. Comparison of left and right ventricular volume measurement using the Simpson's method and the area length method. Eur J Radiol 2007.

[6] Henkens IR, Straten van A, Schalij MJ, et al. Predicting outcome of pulmonary valve replacement in adult tetralogy of Fallot patients. Ann Thorac Surg 2007; 83: 907-11.

[7] Tandri H, Castillo E, Ferrari VA, et al. Magnetic resonance imaging of arrhythmogenic right ventricular dysplasia: sensitivity, specificity, and observer variability of fat detection versus functional analysis of the right ventricle. J Am Coll Cardiol 2006; 48: 227784.

[8] Lelieveldt BP, van der Geest RJ, Lamb HJ, Kayser HW, Reiber JH. Automated observer-independent acquisition of cardiac short-axis MR images: a pilot study. Radiology 2001; 221: 537-42.

[9] Alfakih K, Plein S, Bloomer T, Jones T, Ridgway J, Sivananthan $\mathrm{M}$. Comparison of right ventricular volume measurements between axial and short axis orientation using steady-state free precession magnetic resonance imaging. J Magn Reson Imaging 2003; 18: 2532 .

[10] Alfakih K, Thiele H, Plein S, Bainbridge GJ, Ridgway JP, Sivananthan MU. Comparison of right ventricular volume measurement between segmented k-space gradient-echo and steady-state free precession magnetic resonance imaging. J Magn Reson Imaging 2002; 16: 253-58.

[11] Sechtem U, Pflugfelder PW, Gould RG, Cassidy MM, Higgins CB. Measurement of right and left ventricular volumes in healthy individuals with cine MR imaging. Radiology 1987; 163: 697-702.

[12] Maceira AM, Prasad SK, Khan M, Pennell DJ. Reference right ventricular systolic and diastolic function normalized to age, gender and body surface area from steady-state free precession cardiovascular magnetic resonance. Eur Heart J 2006; 27: 2879-88.

[13] Kacere RD, Pereyra M, Nemeth MA, Muthupillai R, Flamm SD. Quantitative assessment of left ventricular function: steady-state free precession MR imaging with or without sensitivity encoding. Radiology 2005; 235: 1031-35.

[14] Beygui F, Furber A, Delepine S, et al. Routine breath-hold gradient echo MRI-derived right ventricular mass, volumes and function: accuracy, reproducibility and coherence study. Int J Cardiovasc Imaging 2004; 20: 509-16.

[15] Bloomer TN, Plein S, Radjenovic A, et al. Cine MRI using steady state free precession in the radial long axis orientation is a fast ac- curate method for obtaining volumetric data of the left ventricle. J Magn Reson Imaging 2001; 14: 685-92.

[16] Moon JC, Lorenz CH, Francis JM, Smith GC, Pennell DJ. Breathhold FLASH and FISP cardiovascular MR imaging: left ventricular volume differences and reproducibility. Radiology 2002; 223: 78997.

[17] Matheijssen NA, Baur LH, Reiber JH, et al. Assessment of left ventricular volume and mass by cine magnetic resonance imaging in patients with anterior myocardial infarction intra-observer and inter-observer variability on contour detection. Int J Card Imaging 1996; 12: 11-19.

[18] Messroghli DR, Bainbridge GJ, Alfakih K, et al. Assessment of regional left ventricular function: accuracy and reproducibility of positioning standard short-axis sections in cardiac MR imaging. Radiology 2005; 235: 229-36.

[19] Barkhausen J, Ruehm SG, Goyen M, Buck T, Laub G, Debatin JF MR evaluation of ventricular function: true fast imaging with steady-state precession versus fast low-angle shot cine MR imaging: feasibility study. Radiology 2001; 219: 264-69.

[20] Alfakih K, Plein S, Thiele H, Jones T, Ridgway JP, Sivananthan MU. Normal human left and right ventricular dimensions for MRI as assessed by turbo gradient echo and steady-state free precession imaging sequences. J Magn Reson Imaging 2003; 17: 323-29.

[21] Angelini ED, Homma S, Pearson G, Holmes JW, Laine AF. Segmentation of real-time three-dimensional ultrasound for quantification of ventricular function: a clinical study on right and left ventricles. Ultrasound Med Biol 2005; 31: 1143-58.

[22] Pattynama PM, de Roos A, van der Wall EE, Van Voorthuisen AE. Evaluation of cardiac function with magnetic resonance imaging. Am Heart J 1994; 128: 595-07.

[23] Lotjonen JM, Jarvinen VM, Cheong B, et al. Evaluation of cardiac biventricular segmentation from multiaxis MRI data: a multicenter study. J Magn Reson Imaging 2008; 28: 626-36.

[24] Tulevski II, Romkes H, Dodge-Khatami A, et al. Quantitative assessment of the pressure and volume overloaded right ventricle: imaging is a real challenge. Int J Cardiovasc Imaging 2002; 18: 4151 .

[25] Narayan G, Nayak K, Pauly J, Hu B. Single-breathhold, fourdimensional, quantitative assessment of LV and RV function using triggered, real-time, steady-state free precession MRI in heart failure patients. J Magn Reson Imaging 2005; 22: 59-66.

[26] Pattynama PM, Lamb HJ, van der Velde EA, van der Geest RJ, van der Wall EE, de RA. Reproducibility of MRI-derived measurements of right ventricular volumes and myocardial mass. Magn Reson Imaging 1995; 13: 53-63.

[27] Rominger MB, Bachmann GF, Pabst W, Rau WS. Right ventricular volumes and ejection fraction with fast cine MR imaging in breathhold technique: applicability, normal values from 52 volunteers, and evaluation of 325 adult cardiac patients. J Magn Reson Imaging 1999; 10: 908-18.

[28] Straten van A, Vliegen HW, Hazekamp MG, de RA. Right ventricular function late after total repair of tetralogy of Fallot. Eur Radiol 2005; 15: 702-7.

[29] Steen H, Nasir K, Flynn E, et al. Is magnetic resonance imaging the 'reference standard' for cardiac functional assessment? Factors influencing measurement of left ventricular mass and volumes. Clin Res Cardiol 2007; 96: 743-51.

[30] Sandstede J, Lipke C, Beer M, et al. Age- and gender-specific differences in left and right ventricular cardiac function and mass determined by cine magnetic resonance imaging. Eur Radiol 2000; 10: 438-42.

[31] Bavelaar-Croon CD, Kayser HW, van der Wall EE, et al. Left ventricular function: correlation of quantitative gated SPECT and MR imaging over a wide range of values. Radiology 2000; 217: 572-75.

[32] French WJ, Garner D, Hockett D, Laks MM. Ejection fraction derived using dye dilution and angiographic methods. Am Heart J 1982; 104: 104-8.

[33] Canbaz F, Basoglu T, Durna K, Semirgin SU, Canbaz S. Left ventricular aneurysm in the scope of gated perfusion SPECT: accuracy of detection and ejection fraction calculation. Int J Cardiovasc Imaging 2008; 24: 585-96.

[34] Scharhag J, Schneider G, Urhausen A, Rochette V, Kramann B, Kindermann W. Athlete's heart: right and left ventricular mass and function in male endurance athletes and untrained individuals de- 
termined by magnetic resonance imaging. J Am Coll Cardiol 2002; 40: 1856-63.

[35] Pluim BM, Beyerbacht HP, Chin JC, et al. Comparison of echocardiography with magnetic resonance imaging in the assessment of the athlete's heart. Eur Heart J 1997; 18: 1505-13.
[36] Pluim BM, Chin JC, Roos de A, et al. Cardiac anatomy, function and metabolism in elite cyclists assessed by magnetic resonance imaging and spectroscopy. Eur Heart J 1996; 17: 1271-78.

[37] Petersen SE, Hudsmith LE, Robson MD, et al. Sex-specific characteristics of cardiac function, geometry, and mass in young adult elite athletes. J Magn Reson Imaging 2006; 24: 297-303.

Received: May 29, 2008

Revised: September 29, 2008

Accepted: October 29, 2008

(C) Prakken et al.; Licensee Bentham Open.

This is an open access article licensed under the terms of the Creative Commons Attribution Non-Commercial License (http://creativecommons.org/licenses/by-nc/3.0/) which permits unrestricted, non-commercial use, distribution and reproduction in any medium, provided the work is properly cited. 